\title{
Joint preserving surgery for osteoarthritis of the big toe using a cartilage-like implant
}

\begin{abstract}
Dear Editor,
Osteoarthritis of the big toe (hallux rigidus) is a common arthritic condition of the first metatarsophalangeal joint (MTPJ), beginning with stiffness, pain and inflammation. This subsequently progresses to restriction of movements especially dorsiflexion. ${ }^{1}$ Articular surface degeneration and formation of periarticular osteophytes may be seen on radiographs. ${ }^{2}$ Common causes include activity-related repetitive trauma (sports and occupational) and inappropriate footwear. ${ }^{1,3}$ Other factors are direct toe injury, fractures, rheumatoid arthritis, increased age, long proximal phalanx of toe, hypermobile first ray, tarsal coalition, ankylosis of sesamoids to the first metatarsal head, irregular morphology, soft tissue contracture and family history.
\end{abstract}

Treatment options of hallux rigidus remain controversial. These vary from cheilectomy, soft tissue release, first metatarsal osteotomies, arthrodesis, excisional arthroplasty; to hemiarthroplasty and total arthroplasty using different implants such as metal alloy, silastic and ceramic.-6 In our local practice, the commonest surgery for advanced hallux rigidus is arthrodesis. This often results in lower patient satisfaction levels owing to reduction in range of toe movement. Cartiva synthetic cartilage implant (SCI) has been approved by the US Food and Drug Administration (FDA) as an implant for hallux rigidus surgery. The polymer-based polyvinyl alcohol (PVA) implant is recognised as the first synthetic material closest to human joint cartilage. This paper discusses operative technique and clinical outcomes of this innovative joint preservation surgery.

Design and specification of implant. Cartiva SCI is a moulded cylindrical implant composed of PVA and saline. Rigorous biomechanical testing has demonstrated its ability to withstand forces normally subjected on the great toe. ${ }^{7,8}$ Approved by the FDA in 2016 as a PVA hydrogel implant, it is currently used in the US, UK and Europe.

Indications for use. The procedure is reserved for Grade 3 and 4 hallux rigidus where the disease process would be considered moderately severe to severe. ${ }^{9}$ Patients would have undergone a trial period of nonoperative management including analgesia as well as footwear and lifestyle modification. Although this procedure is not considered first line treatment, should patients have had longstanding history of first MTPJ pain lasting several years, then it may be offered at first consultation.

Contraindications. The use of Cartiva SCI is not recommended in cases involving active infection, known/suspected allergy to PVA, gout or rheumatoid arthritis involving first MTPJ and tumour of surrounding bone or tissue. $^{8}$

We present our series of the first 5 cases using this implant. All patients have Grade 3 or 4 hallux rigidus having failed conservative treatment. Pain, function and alignment was graded using an American Orthopaedic Foot and Ankle Society score. ${ }^{10}$

Operative procedure. Surgery is performed with the use of a tourniquet and prophylactic antibiotics. Approach is via a longitudinal dorsal centred over the first MTPJ, followed by dissection down to the joint capsule. The joint is exposed and the periarticular osteophytes are removed. Exposure of the metatarsal head and proximal phalanx is achieved with deep flexion of the joint. A guide wire is inserted into the medullary canal through the centre of the metatarsal head and its position is confirmed with intraoperative imaging. A cannulated drill is then used to core out of the metatarsal head to the desired size (Fig. 1A) for the Cartiva implant. Following the removal of any debris and washing out of the cavity, the implant is carefully inserted using an introducer. The press-fitted final position allows the implant to sit and a $2 \mathrm{~mm}$ protrusion of the top of the implant into the joint is deemed the appropriate height (Fig. 1B). Range of motion of the joint and stability of implant is checked before closing the capsule.

Postoperative care. All patients were discharged on the same day of surgery. Patients were provided an orthopaedic stiff-soled sandal and allowed to weight bear on the operated foot. Early range of motion exercises were started immediately after surgery to prevent joint stiffness. Wound inspection was performed 1 week post-surgery and sutures removed after 2 weeks.

Discussion. Recent local studies of osteoarthritis in larger joints have shown that joint replacement has yielded promising results across different ethnic groups, 


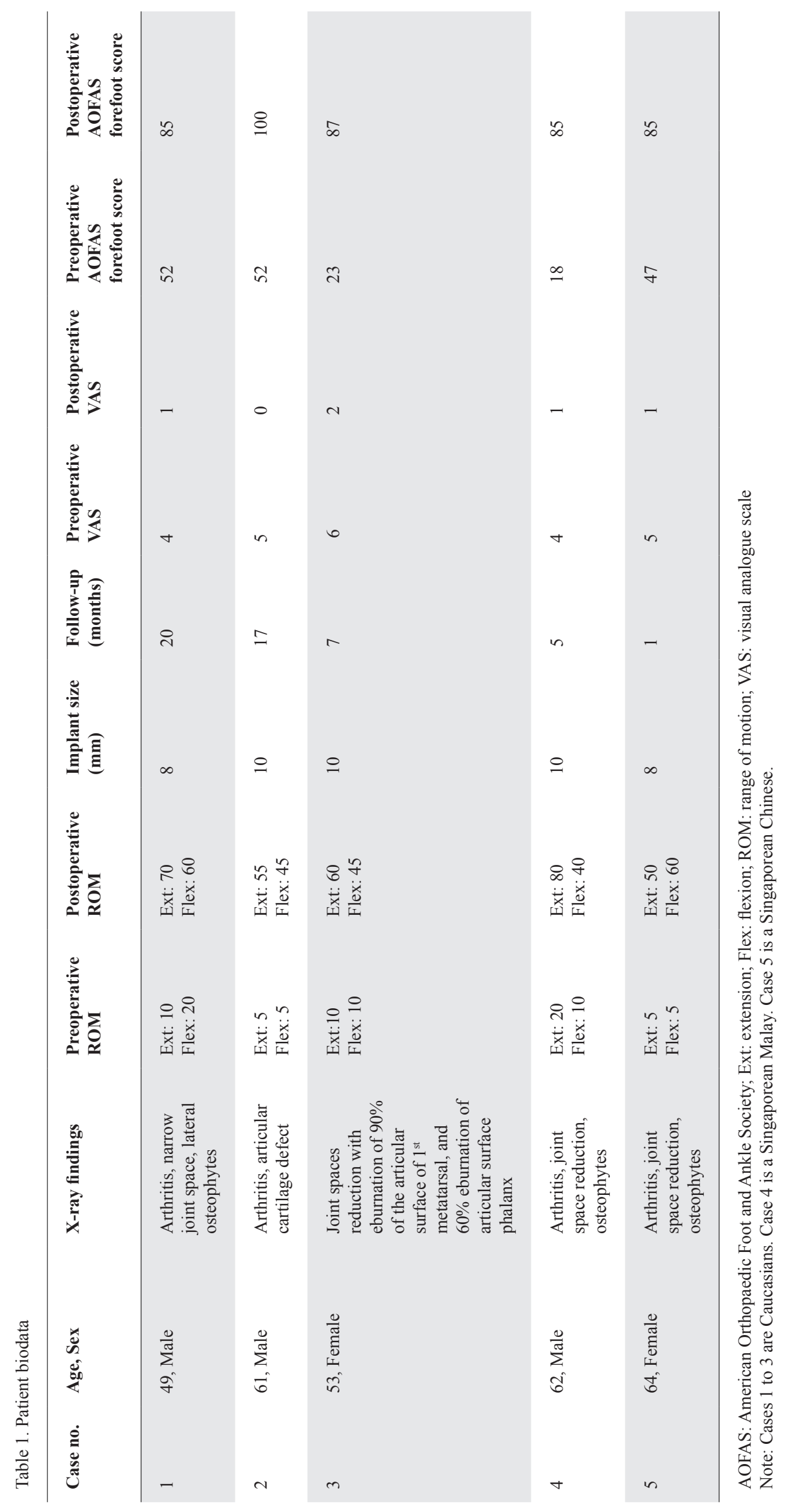




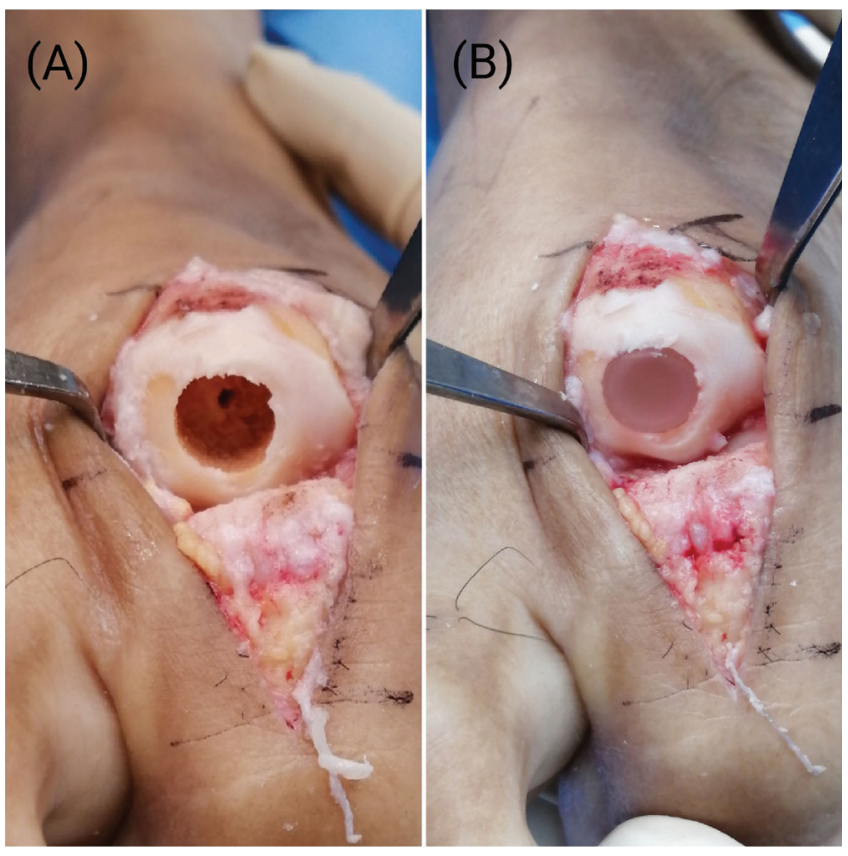

Fig. 1. Intraoperative images of operative procedure. (A) Metatarsal head following excision of surrounding osteophytes and canal preparation. Note the area of cartilage loss. (B) Well-fitted and appropriately sized $(10 \mathrm{~mm}$ diameter) Cartiva implant.

and hence patient satisfaction can be expected. ${ }^{11,12}$ The first MTPJ is also a major site of osteoarthritis that can be treated successfully with joint replacement surgery. ${ }^{1,9,11}$ Our paper highlights good patient satisfaction from different ethnic backgrounds (Table 1), in accordance with the local studies. ${ }^{11,12}$

Treatment modalities for the hallux rigidus are controversial and many options have been described in management of the disease. Non-surgical management of stage 1 and stage 2 hallux rigidus may provide some relief to the patients. ${ }^{9}$ For advanced stage, surgical intervention is needed to reduce pain and improve functional stability. The type of surgery depends upon the stage of hallux rigidus. ${ }^{13}$ Cheilectomy surgical procedure (removal of osteophytes and joint debridement) is usually advocated to treat mild hallux rigidus. ${ }^{1}$ Closing wedge osteotomy (dorsal) can be performed for the phalanx to improve functional stability. ${ }^{14}$ Arthrodesis, accepted as the gold standard technique for grade 3 and 4 hallux rigidus, provides pain relief but sacrifices joint movements. ${ }^{4,8}$

The biggest benefit of joint replacement surgery is preservation of joint movements and a shorter rehabilitation with immediate mobilisation. Although initial results of other implants were promising, they subsequently led to failure. Silastic (silicon rubber) resulted in osteolysis and inflammatory immune reaction causing bony resorption and implant loosening. ${ }^{7}$
Metal alloy and ceramic implants failed because of relative hardness of the material versus bone, causing wear within the bony canal. ${ }^{15}$

We have presented promising results following surgery for hallux rigidus using this new implant. No complications were reported in our series. Serial radiological and clinical examination found no evidence of failure and implant loosening.

Studies in the UK and North America show similar results in favour of first MTPJ arthroplasty using Cartiva implant. ${ }^{12}$

We believe that for Singapore's population, joint replacement can be the "new gold standard" for moderate to advanced stages of hallux rigidus. This can benefit patients who are active and wish to have their toe movements preserved; and women who want to continue wearing high heels. ${ }^{16}$

\section{REFERENCES}

1. Shereff MJ, Baumhauer JF. Hallux rigidus and osteoarthrosis of the first metatarsophalangeal joint. J Bone Joint Surg Am 1998;80:898-908.

2. Harisboure A, Joveniaux P, Madi K, et al. The Valenti technique in the treatment of hallux rigidus. Orthop Traumatol Surg Res 2009;95:202-9.

3. Cannavò L, Costarella L, Pavone V, et al. Arthrodesis and Hemiarthroplasty: Different Techniques in the Treatment of Hallux Rigidus-Surgery and Postoperative Rehabilitation. J Funct Morphol Kinesiol 2016;1:102-8.

4. Bennett GL, Sabetta J. First metatarsalphalangeal joint arthrodesis: evaluation of plate and screw fixation. Foot Ankle Int 2009;30:752-7.

5. Brage ME, Ball ST. Surgical options for salvage of end-stage hallux rigidus. Foot Ankle Clin 2002;7:49-73.

6. Chee YH, Clement N, Ahmed I, et al. Functional outcomes following ceramic total joint replacement for hallux rigidus. Foot Ankle Surg 2011;17:8-12.

7. Ingham E, Fisher J. Biological reactions to wear debris in total joint replacement. Proc Inst Mech Eng H 2000;214:21-37

8. Baumhauer JF, Singh D, Glazebrook M. Prospective, randomized, multi-centered clinical trial assessing safety and efficacy of a synthetic cartilage implant versus first metatarsophalangeal arthrodesis in advanced hallux rigidus. Foot Ankle Int 2016;37:457-69.

9. Lam A, Chan JJ, Surace MF, et al. Hallux rigidus: How do I approach it? World J Orthop 2017;8:364-71.

10. Kitaoka HB, Alexander IJ, Adelaar RS, et al. Clinical rating system for the ankle-hindfoot, midfoot, hallux, and lesser toes. Foot Ankle Int 1994;15:349-53.

11. Shah NZ, Malhotra R, Hong CC, et al. Ethnic Differences in Preoperative Patient Characteristics and Postoperative Functional Outcomes after Total Knee Arthroplasty among Chinese, Malays and Indians. Ann Acad Med Singap 2018;47:201-5.

12. Huang Y, Lee M, Chong HC, et al. Reasons and Factors Behind Post-Total Knee Arthroplasty Dissatisfaction in an Asian Population. Ann Acad Med Singap 2017;46:303-9. 
13. Coughlin MJ, Shurnas PS. Hallux rigidus. Grading and long-term results of operative treatment. J Bone Joint Surg Am 2003; $85: 2072-88$

14. O'Malley MJ, Basran HS, Gu Y, et al. Treatment of advanced stages of hallux rigidus with cheilectomy and phalangeal osteotomy. J Bone Joint Surg Am 2013;95:606-10.

15. Cracchiolo A, Weltmer JB, Lian G, et al. Arthroplasty of the first metatarsophalangeal joint with a double-stem silicone implant. Results in patients who have degenerative joint disease failure of previous operations, or rheumatoid arthritis. J Bone Joint Surg Am 1992;74:552-63.

16. Baker MI, Walsh SP, Schwartz Z, et al. A review of polyvinyl alcohol and its uses in cartilage and orthopaedic applications. J Biomed Mater Res B Appl Biomater 2012;100:1451-7.
Yu Han $\underline{\text { Chee }}{ }^{1}$ FRCS Ed (Tr\&Orth), Ishwar Meena ${ }^{1} M S$ (Orth), Sean JK Lee ${ }^{1}{ }_{M B B S}$

${ }^{1}$ Department of Orthopaedic Surgery, National University Hospital, Singapore

Correspondence: Dr Yu Han Chee, Division of Foot \& Ankle Surgery, University Orthopaedics, Hand \& Reconstructive Microsurgery Cluster, National University Health System, NUHS Tower Block,

1E Kent Ridge Road, Level 11, Singapore 119228.

Email: yu_han_chee@nuhs.edu.sg 